\title{
Research on Fuzzy Theory-Based Government Promotion to Public Sports Service Development
}

\author{
Dahai Liu*
}

Department of Physical Education, Huaihai Institute of Technology, Lianyungang 222005, Jiangsu, China

\begin{abstract}
The Chinese government functions presentation in sports, many of them have been rapidly developed, the paper according to status that Chinese government promotes to sports public service development, applies fuzzy mathematics to represent some factors set into people's consciousness. By establishing attributes scale on one object, it carries on fuzzy mathematical analysis of one object, in China's enhanced physical ability exercises state budget, it mainly invests in sports facilities, sports relative staff cultivation, organizing all kinds of sports to cultivate, sports relevant undertakings revitalization these four aspects, and meanwhile the paper also according to the four aspects, it makes fuzzy comprehensive evaluation, gets that Chinese government such four aspects sports construction research. By fuzzy comprehensive evaluation, the paper gets fuzzy comprehensive evaluation value, it indicates located indicator range is in the score interval of $80-90$, therefore it can illustrate that Chinese government plays crucial roles in promoting public sports service development, which should attract higher attention.
\end{abstract}

Keywords: Factor analysis, fuzzy evaluation, public sports service.

\section{INTRODUCTION}

From the medium-term of 60 s to 80 s, Chinese economy has been rapidly developed, Chinese government carries out social sports development, it lets Chinese tertiary industry proportion to greatly increase, and in order to propel to sports development, the Chinese government drives every sector to vigorously carry out sports development, and revitalize national sports $[1,2]$.

With Chinese opening-up and reform, Chinese sports public service field has been hugely developed, due to implementation of Chinese civil public sports right, Chinese government supplying mode on publics sports service tends to regular, and speed up the coordination of all aspects and sports public service industry relationships [3].

Fuzzy mathematics is a theoretical system that is formed by fuzzy set and fuzzy logic, fuzzy mathematics is applied in pattern recognition and artificial intelligence, as a relative brand new discipline, fuzzy mathematics represent some factors set into people's consciousness [4]. By establishing attributes scale on one object, it carries on fuzzy mathematical analysis of one object, firstly the object should have fuzziness or uncertainty, and researched objects have multiple influence factors restriction, therefore the paper researches on Chinese government promotion to public sports service development according to fuzzy comprehensive evaluation.

\section{CHINESE SPORTS PUBLIC SERVICE STATUS ANALYSIS}

Chinese public sports service facilities budget after year 1971 , occupied sports facilities budget total proportion is increasing by year, as Table $\mathbf{1}$ shows.

As Table 2 shows, China enhances physical ability exercise state budget, carries out state investment in apparatus facility, department revitalization, organizing cultivation, coach cultivation four aspects.

By Table 1 showed data, we get total amount change graph with time increasing, as Fig. (1) shows.

In Fig. (1), it is clearly indicated that Chinese government state budget on enhancing physical ability exercise is in the yearly rising trend.

\subsection{Fuzzy Comprehensive Evaluation Model}

Fuzzy comprehensive evaluation model is fit for multiple factors uncertain fuzzy calculation, the paper utilizes fuzzy comprehensive evaluation, and steps are as following:

At first the paper establishes factor set $U$, $U=\left(\begin{array}{llll}U_{1} & U_{2} & \cdots & U_{k}\end{array}\right)$

(2) Secondly establish evaluation set $V$ (assessment set) $V$,

(3) Establish evaluation matrix fuzzy mapping from $U$ to $V$, it gets fuzzy relation as following matrix shows, $R=\left[\begin{array}{cccc}r_{11} & r_{12} & \cdots & r_{1 n} \\ r_{21} & r_{22} & \cdots & r_{2 n} \\ \vdots & \vdots & & \vdots \\ r_{m 1} & r_{m 2} & \cdots & r_{m n}\end{array}\right]$

2015 Bentham Open 
Table 1. Year 1971 to 2004 government to sports facilities budget and public sports facilities budget.

\begin{tabular}{|c|c|c|}
\hline Time & Sports Facilities Budget (Hundred Million) & Public Sports Facilities Budget (Hundred Million) \\
\hline Year 1971 & 35.28945 & 7.07500 \\
\hline Year 1972 & 41.945456 & 17.456412 \\
\hline Year 1973 & 58.8954 & 21.18850 \\
\hline Year 1974 & 89.31950 & 37.96750 \\
\hline Year 1988 & 169.46429 & 66.12856 \\
\hline Year 1989 & 185.45864 & 78.45875 \\
\hline Year 1990 & 186.45867 & 80.45674 \\
\hline Year 1991 & 189.45678 & 78.78156 \\
\hline Year 1992 & 194.35421 & 89.48564 \\
\hline Year 1995 & 253.34455 & 153.57016 \\
\hline Year 1996 & 265.45784 & 162.45678 \\
\hline Year 1997 & 285.88654 & 98.748564 \\
\hline Year 2000 & 480.45674 & ---- \\
\hline Year 2001 & 5380.37463 & 86.4554 \\
\hline Year 2002 & 3873.56447 & 12.84756 \\
\hline Year 2003 & 358.84719 & 11.45787 \\
\hline Year 2004 & 306.53946 & 10.45786 \\
\hline
\end{tabular}

Table 2. Chinese enhancement of physical ability exercises state budge statistics in 1996.

\begin{tabular}{|c|c|c|c|c|c|c|}
\hline Year & $\begin{array}{c}\text { Apparatus Facil- } \\
\text { ity }\end{array}$ & $\begin{array}{c}\text { Department Revi- } \\
\text { talization }\end{array}$ & $\begin{array}{l}\text { Organizing } \\
\text { Cultivation }\end{array}$ & $\begin{array}{c}\text { Coach } \\
\text { Cultivation }\end{array}$ & Total Amount & GDP $(\%)$ \\
\hline 1985 & 1604 & 710 & 70 & 6 & 2309 & 0.071 \\
\hline 1986 & 1455 & 541 & 75 & 6 & 2770 & 0.071 \\
\hline 1987 & 1441 & 965 & 74 & 6 & 2598 & 0.075 \\
\hline 1988 & 1707 & 986 & 72 & 9 & 2921 & 0.081 \\
\hline 1989 & 1777 & 1156 & 74 & 12 & 3081 & 0.084 \\
\hline 1990 & 1903 & 1193 & 85 & 28 & 3562 & 0.085 \\
\hline 1991 & 2184 & 1276 & 91 & 30 & 3978 & 0.087 \\
\hline 1992 & 2363 & 1456 & 98 & 72 & 3964 & 0.085 \\
\hline 1993 & 2670 & 1345 & 100 & 80 & 4336 & 0.093 \\
\hline 1994 & 2624 & 1786 & 1000 & 73 & 4456 & 0.095 \\
\hline 1995 & 2788 & 1535 & 98 & 95 & 4689 & 0.094 \\
\hline
\end{tabular}

(4) Establish weight set, $A=\left(a_{1}, a_{2}, \cdots, a_{n}\right)$, it meets conditions: $\sum_{i=1}^{n} a_{i}=1 \quad a_{i} \geq 0$
(5)Fuzzy relation $R$ every line will reflect the line influence factors to object judgment degree, meanwhile, $R$ every column will reflect the column influence factors to object judgment degree. 


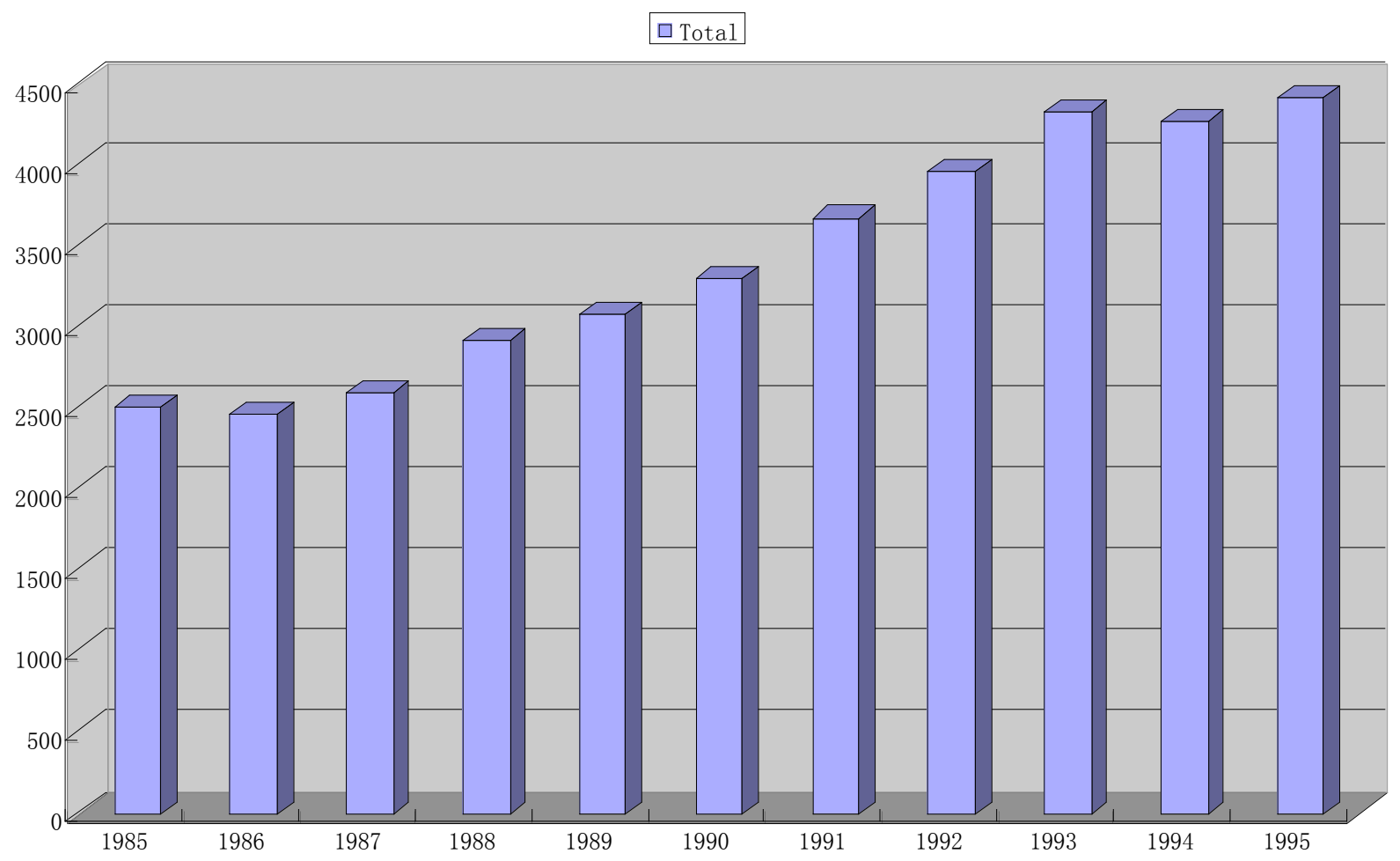

Fig. (1). Total amount change graph with time increasing.

$$
\sum_{i=1}^{n} r_{i j} \quad j=1,2,3, \cdots, m
$$

Secondly carry out following calculation:

$$
\begin{aligned}
B & =A \cdot R \\
& =\left(a_{1}, a_{2}, a_{3}, \cdots, a_{n}\right) \cdot\left[\begin{array}{cccc}
r_{11} & r_{12} & \cdots & r_{1 n} \\
r_{21} & r_{22} & \cdots & r_{2 n} \\
\vdots & \vdots & & \vdots \\
r_{m 1} & r_{m 2} & \cdots & r_{m n}
\end{array}\right] \\
& =\left(b_{1}, b_{2}, b_{3}, \cdots, b_{n}\right)
\end{aligned}
$$

In $V$, fuzzy combination is evaluation set $B$. Based on above described facts, actual fuzzy comprehensive evaluation obtained simple change model is as Fig. (2) shows:

With Fig. (2) marketed contents, it gets fuzzy comprehensive evaluation change model, and can establish corresponding every factor grade evaluation transformation function, evaluation factors $\mathrm{u} 1, \mathrm{u} 2, \mathrm{u} 3, \mathrm{u} 4, \mathrm{u} 5$ membership functions can be expressed as formula(1)、(2)、(3) shows :

$$
u_{v 1}\left(u_{1}\right)=\left\{\begin{array}{cc}
0.5\left(1+\frac{u_{i}-k_{1}}{u_{i}-k_{2}}\right), & u_{i} \geq k_{1} \\
0.5\left(1-\frac{k_{1}-u_{i}}{k_{1}-k_{2}}\right), & k_{2} \leq u_{i}<k_{1} \\
0 \quad, & u_{i}<k_{2}
\end{array}\right.
$$

$$
u_{v 2}\left(u_{1}\right)=\left\{\begin{array}{lr}
0.5\left(1-\frac{u_{i}-k_{1}}{u_{i}-k_{2}}\right), & u_{i} \geq k_{1} \\
0.5\left(1+\frac{k_{1}-u_{i}}{k_{1}-k_{2}}\right), & k_{2} \leq u_{i}<k_{1} \\
0.5\left(1-\frac{u_{i}-k_{3}}{k_{2}-k_{3}}\right), & k_{3} \leq u_{i}<k_{2} \\
0.5\left(1-\frac{k_{3}-u_{i}}{k_{2}-u_{i}}\right), & u_{i}<k_{3}
\end{array}\right.
$$

$$
u_{v 1}\left(u_{1}\right)=\left\{\begin{array}{cc}
0, & u_{i} \geq k_{2} \\
0.5\left(1-\frac{k_{1}-u_{i}}{k_{2}-k_{3}}\right), & k_{3} \leq u_{i}<k_{2} \\
0.5\left(1+\frac{k_{3}-u_{i}}{k_{2}-u_{i}}\right), & u_{i}<k_{3}
\end{array}\right.
$$

\subsection{Combine with fuzzy Evaluation Model to Evaluate}

By above model principle, it establishes factor set $U$, from which $U=\left(\begin{array}{llll}U_{1} & U_{2} & U_{3} & U_{4}\end{array}\right)$. Among them, sports facilities $U_{1}$, sports relative staff cultivation $U_{2}$, organizing all kinds of sports to cultivate $U_{3}$, sports relevant undertakings revitalization $U_{4}$, it gets Table 3 . The paper establishes small factors set in the four important factors.

By Table 3 listed factors, it gets evaluation set. 
Table 3. Chinese sports undertakings evaluation indicator system.

\begin{tabular}{|c|c|c|c|}
\hline Facilities $U_{1}$ & Staff Cultivation $U_{2}$ & Organizing Cultivation $U_{3}$ & Undertakings Revitalization $U_{4}$ \\
\hline \hline Facility introduction $u_{11}$ & Coaches cultivation $u_{21}$ & Competition $u_{31}$ & Undertakings introduction $u_{41}$ \\
\hline Facilities maintenance $u_{12}$ & $\begin{array}{c}\text { Teaching and administrative staff } \\
\text { cultivation } u_{22}\end{array}$ & Activity $u_{32}$ & Undertakings development $u_{42}$ \\
\hline $\begin{array}{c}\text { Competition facilities construction } \\
u_{13}\end{array}$ & Foreign coaches introduction $u_{23}$ & Lecturing $u_{33}$ & $\begin{array}{c}\text { Traditional undertakings revitaliza- } \\
\text { tion } u_{43}\end{array}$ \\
\hline $\begin{array}{c}\text { Daily facilities construction } u_{14} \\
\text { Apparatus maintenance and chang- } \\
\text { ing } u_{15}\end{array}$ & Cultivation expense $u_{24}$ & Overseas tour $u_{34}$ & \\
\hline
\end{tabular}

Table 4. Four kinds of factors importance degree ranking statistics.

\begin{tabular}{|c|c|c|c|c|}
\hline Classification & Rank1 & Rank 2 & Rank 3 & Rank 4 \\
\hline \hline Facilities $U_{1}$ & 23 & 7 & 15 & 18 \\
\hline Staff cultivation $U_{2}$ & 0 & 0 & 13 & 12 \\
\hline Organizing cultivation $U_{3}$ & 0 & 9 & 9 & 0 \\
\hline Undertakings revitalization $U_{4}$ & 3 & 21 & & 0 \\
\hline
\end{tabular}

$$
\begin{aligned}
& U_{1}=\left\{u_{11}, u_{12}, u_{13}, u_{14}\right\} \\
& U_{2}=\left\{u_{21}, u_{22}, u_{23}, u_{24}, u_{25}\right\} \\
& U_{3}=\left\{u_{31}, u_{32}, u_{33}\right\} \\
& U_{4}=\left\{u_{41}, u_{42}, u_{43}, u_{44}\right\}
\end{aligned}
$$

By collecting data and analyzing, it gets four kinds of factors importance ranking statistics, as Table $\mathbf{4}$ shows.

By Table 4 sorting, it gets sports facilities $U_{1}$, sports relative staff cultivation $U_{2}$, organizing all kinds of sports to cultivate $U_{3}$, sports relevant undertakings revitalization $U_{4}$ four aspects' rank matrix.

$$
U_{2}=\{23,7,4,0\} ; \quad U_{2}=\{7,18,80\} ; \quad U_{3}=\{0,9,13,12\} ;
$$

$U_{4}=\{3,0,9,21\}$

Obtained weighted vector from rank 1 to rank 2

$$
\begin{aligned}
& \beta=\left\{\beta_{1}, \beta_{2}, \beta_{3}, \beta_{4}\right\}=\{0.4,0.3,0.2,0.1\} \\
& U_{i}^{*}=U_{i} \cdot \beta^{T} \\
& U_{1}^{*}=14, U_{2}^{*}=9.4, U_{3}^{*}=4, U_{4}^{*}=5.6
\end{aligned}
$$

The paper takes normalization processing $U_{1}^{*}=0.35$, $U_{2}^{*}=0.3, U_{3}^{*}=0.2, U_{4}^{*}=0.15$

It gets: $\bar{A}=\left(\begin{array}{llll}0.35 & 0.3 & 0.2 & 0.15\end{array}\right)$
The paper establishes remarks membership, as Table 5 show.

The paper through Chinese sports each indicator obtained evaluation in sports facilities, sports relative staff cultivation, organizing all kinds of sports to cultivate, sports relevant undertakings revitalization all aspects, it gets Table 6.

By above model, it gets single layer indicator weight factor fuzzy set is:

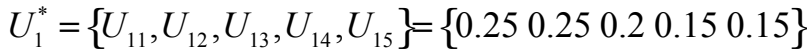

$$
\begin{aligned}
& U_{2}^{*}=\left\{U_{21}, U_{22}, U_{23}, U_{24}\right\}=\left\{\begin{array}{lllll}
0.54 & 0.1 & 0.24 & 0.14
\end{array}\right\} \\
& U_{1}^{*}=\left\{U_{31}, U_{32}, U_{33}, U_{34}\right\}=\left\{\begin{array}{llll}
0.4 & 0.3 & 0.1 & 0.2
\end{array}\right\} \\
& U_{1}^{*}=\left\{U_{41}, U_{42}, U_{43}\right\}=\left\{\begin{array}{lll}
0.3 & 0.4 & 0.3
\end{array}\right\}
\end{aligned}
$$

By Table 5, and combine with Table $\mathbf{3}$ remarks membership, the paper gets sports facilities $U_{1}$, sports relative staff cultivation $U_{2}$, organizing all kinds of sports to cultivate $U_{3}$, sports relevant undertakings revitalization $U_{4}$ each aspect evaluation set

$$
\text { Sports facilities } U_{1}=\left(\begin{array}{cccc}
0 & 0 & 0.05 & 0.95 \\
0 & 0 & 0.05 & 0.95 \\
0 & 0.05 & 0.95 & 0.05 \\
0 & 0.05 & 0.95 & 0.05 \\
0 & 0.05 & 0.95 & 0.05
\end{array}\right)
$$


Table 5. Remarks membership.

\begin{tabular}{|c|c|c|c|c|}
\hline \multirow{2}{*}{ Evaluation Way } & \multicolumn{5}{|c|}{ Set Scores Interval } \\
\cline { 2 - 5 } & $\mathbf{0 - 6 0}$ & $\mathbf{6 0 - 8 0}$ & $\mathbf{8 0 - 9 0}$ & $\mathbf{9 0 - 1 0 0}$ \\
\hline \hline Very good & 0 & 0 & 0.05 & 0.95 \\
\hline Good & 0 & 0.05 & 0.9 & 0.05 \\
\hline Normal & 0.05 & 0.9 & 0.05 & 0 \\
\hline Bad & 0.95 & 0.05 & 0 & 0 \\
\hline
\end{tabular}

Table 6. Chinese sports each indicator obtained evaluation value.

\begin{tabular}{|c|c|c|c|}
\hline Each Layer Indicator & Evaluation Value & Each Layer Indicator & Evaluation Value \\
\hline \hline Facility introduction $u_{11}$ & Very good & Competition $u_{31}$ & Very good \\
\hline Facilities maintenance $u_{12}$ & Very good & Activity $u_{32}$ & Gecturing $u_{33}$ \\
\hline $\begin{array}{c}\text { Competition facilities construction } \\
u_{13}\end{array}$ & Normal & Overseas tour $u_{34}$ & Nood \\
\hline $\begin{array}{c}\text { Daily facilities construction } u_{14} \\
\text { Apparatus maintenance and chang- } \\
\text { ing } u_{15}\end{array}$ & Normal & Undertakings introduction $u_{41}$ & Good \\
\hline $\begin{array}{c}\text { Coaches cultivation } u_{21} \\
\text { Teaching and administrative staff } \\
\text { cultivation } u_{22}\end{array}$ & Very good & Undertakings development $u_{42}$ & Very good \\
\hline $\begin{array}{c}\text { Foreign coaches introduction } u_{23} \\
\text { Cultivation expense } u_{24}\end{array}$ & Very good & Traditional undertakings revitaliza- & Normal \\
\hline
\end{tabular}

Sports relative staff cultivation $U_{2}=\left(\begin{array}{cccc}0 & 0 & 0.05 & 0.95 \\ 0 & 0 & 0.05 & 0.95 \\ 0 & 0 & 0.05 & 0.95 \\ 0 & 0.05 & 0.9 & 0.05\end{array}\right)$

Organizing all kinds of sports to cultivate $U_{3}=\left(\begin{array}{cccc}0 & 0 & 0.05 & 0.95 \\ 0 & 0.05 & 0.9 & 0.05 \\ 0 & 0.05 & 0.9 & 0.05 \\ 0.05 & 0.9 & 0.05 & 0\end{array}\right)$

Sports relevant undertakings revitalization $U_{4}=\left(\begin{array}{cccc}0 & 0 & 0.05 & 0.95 \\ 0 & 0.05 & 0.9 & 0.05 \\ 0 & 0.05 & 0.9 & 0.05\end{array}\right)$

For above evaluation sets, carry on following calculation: $B_{i}=A_{i} \cdot R_{i}$

Make normalization processing with obtained $B_{i}$, it gets fuzzy evaluation matrix.

$$
\bar{B}=\left(\begin{array}{l}
B_{1} \\
B_{2} \\
B_{3} \\
B_{4}
\end{array}\right)=\left(\begin{array}{cccc}
0.07 & 0.27 & 0.23 & 0.43 \\
0 & 0.1 & 0.7 & 0.5 \\
0.08 & 0.16 & 0.28 & 0.28 \\
0.14 & 0.2 & 0.3 & 0.36
\end{array}\right)
$$

It gets comprehensive evaluation value: $Z=U^{*} \cdot B=\left(\begin{array}{llll}0.39 & 0.06 & 0.25 & 0.30\end{array}\right)$

\section{CONCLUSION}

Fuzzy mathematics is from people recognition on external world, due to suffer numerous factors influences, human recognized things are fuzzy. By fuzzy mathematics, it analyzes Chinese government promotion to public sports service development. By analysis, it gets that Chinese government in sports facilities $U_{1}$, sports relative staff cultivation $U_{2}$, organizing all kinds of sports to cultivate $U_{3}$, sports relevant undertakings revitalization $U_{4}$ four aspects evaluation value is : $Z=\left(\begin{array}{llll}0.39 & 0.06 & 0.25 & 0.30\end{array}\right)$. By result indication, on a whole, Chinese government plays crucial roles in promoting public sports service development, 
but in some aspects, such as overseas tour, apparatus maintenance and changing, traditional undertakings revitalization and other aspects, it needs to be further improved.

\section{CONFLICT OF INTEREST}

The author confirms that this article content has no conflict of interest.

\section{ACKNOWLEDGEMENTS}

Declared none.

\section{REFERENCES}

[1] C. Pei-You, and L. Xing, "Fuzzy comprehensive evaluation algorithm improvement," J. Heilongjiang. Inst. Technol., vol. 16, no. 6, pp. 396-399, 2006

[2] L. De-Chun, "Enlightenment of Chinese government social sports revitalization policies promotion on Chinese national fitness development," J. Shenyang Inst. Phys. Educ., no. 2, 2002.

[3] L. Xi-Lin, W. Lai-Gui, Z. Kui, and L. Ling, "Fuzzy comprehensive evaluation method improvement and application in mine water's quality of water evaluation," J. Water Reso. Water Eng., vol. 20, no. 5 , pp. 5-8, 2009.

[4] R. Hua, and C. Hong, "China sports facilities construction and development countermeasures comparative research," J. Shandong Sport. Inst., no. 6, pp. 18-20, 2006.

Received: June 10, 2015

Revised: July 29, 2015

Accepted: August 15, 2015

(C) Dahai Liu; Licensee Bentham Open.

This is an open access article licensed under the terms of the (https://creativecommons.org/licenses/by/4.0/legalcode), which permits unrestricted, noncommercial use, distribution and reproduction in any medium, provided the work is properly cited. 